\title{
Die Europäische Union, Kirchen und Religionen: Rückblicke auf eine Kontroverse
}

\author{
Heinrich Schneider*
}

\section{„Gott und die Kirchen - hinein in die Verfassung!“ - ein erledigtes Thema?}

Der Vertragsentwurf über eine ,Verfassung für Europa (VVE) ist Vergangenheit; immerhin kam es seither zur Einigung über den ,Vertrag von Lissabon', nun hofft man auf die Ratifizierung. Inwieweit der Ertrag der Verfassungsarbeit über die Krise hinweggerettet wurde war umstritten - weil die Kriterien dafür nicht selbstverständlich sind, aber auch, weil vom Urteil darüber für manche Länder die Nötigung zu einem Referendum abhängt.

Eine Neuerung ist dabei kaum erörtert worden: Der Artikel über den Status der Kirchen, religiösen oder weltanschaulichen Gemeinschaften und Vereinigungen, sowie über den von der Union mit ihnen zu pflegenden Dialog wurde nicht mehr thematisiert. Ganz selbstverständlich ist das nicht, denn die Bestimmung bekräftigt zumindest implizit den Charakter der Union als ein Gemeinwesen, dessen Raison nicht auf „Systemintegration“ (im Begriffsverständnis von Jürgen Habermas) ${ }^{1}$ reduziert werden kann. Wozu sollte der institutionalisierte Dialog mit Religions- und Weltanschauungsgemeinschaften gut sein, wenn die Union nur dem besseren Interdependenzmanagement und der Interessenbündelung dienen, wenn sie nur ein (wenn auch nicht mehr auf die Wirtschaftsintegration beschränkter) Zweckverband sein soll, und nicht auch eine politisch verfasste Wertegemeinschaft? Insofern hätten jene, die die ,Polity'-Qualität des Staatenverbunds zurechtstutzen wollten, ähnlich wie die Liquidierung des Artikels über die Symbole der Union auch die des sogenannten Kirchenartikels fordern können.

Er blieb erhalten. ,Causa finita', möchte man meinen. ,Die Religionen und die Europäische Union" - das Thema ist wohl bis auf Weiteres von der Tagesordnung abgesetzt. Soll man es in Frieden ruhen lassen?

Da das Thema in der Phase der Verfassungsarbeit die Gemüter da und dort in erstaunlichem Maße bewegt hat, könnte eine Nachbetrachtung nützlich sein.

\section{Die Religionsgemeinschaften und ihre Anliegen - ein Element der Europapolitik}

In Erinnerung wird am ehesten noch ein kirchliches Anliegen geblieben sein, für das sich - neben höchsten katholischen Würdenträgern, mit dem Papst an der Spitze - auch etliche Mitgliedstaaten (nicht nur Polen) engagierten, wohingegen andere sich massiv widersetzten: Neben dem sogenannten Gottesbezug in der Verfassungspräambel ging es um die Betonung des christlichen Erbes Europas, oder um das Bekenntnis zu „,christlichen Werten“. Viele meinten: nun erst, mit der Debatte darüber, hätte die viel diskutierte „Rückkehr der Religion in die Politik“" endlich auch die Europäische Union erreicht.

Ganz richtig ist das nicht, auch wenn lange Zeit hindurch Verknüpfungen zwischen Europapolitik und Religionsangelegenheiten wenig Aufmerksamkeit fanden. Immerhin brachten Gegner der Integration in deren Frühzeit ihre Abneigung auf die Formel „Vom Vatikan gezeugt - von Washington gesäugt“; die Sympathiebekundungen der römisch-katholischen

Prof. Dr. Heinrich Schneider, Universität Wien; Institut für Europäische Politik, Berlin.

1 Jürgen Habermas: Theorie des kommunikativen Handelns, Band 1 und 2, Frankfurt am Main 1981. 
Kirche für die europäische Einigung waren tatsächlich eindeutig und zahlreich. ${ }^{2}$ Nur selten berichteten die Medien über spektakuläre Vorgänge. Einer davon war der ,Fall Buttiglione“; damals sprachen manche Kommentatoren von einem „Kulturkampf“; namhafte Europapolitiker von einem „Kreuzzug“: Sollte es ihn wirklich gegeben haben, dann war er partei- und verfassungspolitisch instrumentalisiert worden (im Machtkampf zwischen Parlament und Kommission, wobei einige Akteure auch dem italienischen Regierungschef Silvio Berlusconi eins auswischen wollten). Doch hieß es auch: nun sei endlich einmal der oft camouflierte Widerspruch zwischen kirchlichen Glaubenslehren und den Grundsätzen des liberalen Rechtsstaats offenkundig geworden. ${ }^{3}$

Freilich: das war eine Episode. Das Ringen um Präambelformeln und um die besondere Würdigung der Kirchen, Religions- und Weltanschauungsgemeinschaften, die in Anbetracht ihres „,besonderen Beitrags“ zur europäischen Sache zu Partnern eines speziellen Dialogs werden sollten (unterschieden von dem mit den ,repräsentativen Verbänden“ und mit der „Zivilgesellschaft“), hatte grundsätzlicheren Charakter.

\section{Die ,Invocatio Dei', ihre Alternativen - und die Motivhintergründe}

Was die Präambel betrifft, so war das Verlangen nach einem Präambelsatz, in dem von Gott die Rede sein sollte, am anspruchsvollsten. Dafür wurde oft das Etikett ,invocatio Dei “ verwendet - das war unkorrekt: eine ,Anrufung Gottes', wie sie etwa im Eröffnungssatz der Verfassung Irlands ausgesprochen wird, ${ }^{4}$ hatte niemand gewünscht. In einer echten ,invocatio“ wäre Gott der Adressat eines Glaubensbekenntnisses im Namen der eigentlichen Inhaber der verfassunggebenden Gewalt - und/oder der angerufene Bürge eines Verpflichtungsgelöbnisses auf die Verfassung (oder auf bestimmte zentrale Inhalte), vergleichbar einer Eidesformel: „So wahr uns Gott helfe!“‘.

In einer extrem weitgehenden Auslegung könnte das womöglich als eine Selbstweihe des Volkes an Gott gelten; freilich nicht auch schon als Sakralisierung der Verfassung als solcher, sodass sie in Gottes Hand gegeben und damit menschlicher Verfügbarkeit entzogen würde (dies schlösse eine Absage an das moderne, säkulare Verständnis von, Volkssouveränität‘ ein). Wohl aber könnte man eine solche Formel als eine Überantwortung der konstitutionellen Ordnung an das Regiment und/oder die Gnade Gottes verstehen.

Andere Bewandtnisse hätte es mit einer ,nominatio“ (oder , enuntiatio') Gottes, also mit einem Gott erwähnenden Satz ohne Anrufungscharakter; unterschiedliche Varianten wären möglich. Eine bietet das Grundgesetz: das „Deutsche Volk“ hat es sich ,im Bewußtsein seiner Verantwortung vor Gott und den Menschen“ gegeben. Eine Alternative wäre der Hinweis auf die Gottgläubigkeit derer, in deren Namen die Verfassung gegeben wird, oder doch

2 Siehe z.B. Heinrich Schneider: Die Europäische Einigung als Thema der Katholischen Kirche, in: Peter-Christian Müller-Graff/Heinrich Schneider (Hrsg.): Kirchen und Religionsgemeinschaften in der Europäischen Union, Baden-Baden 2003, S. 73ff. Die Kirchen der Reformation und die Orthodoxie taten sich schwerer.

3 Der Satz stammte, wie auch das Wort vom Kreuzzug, von Kommissionsvizepräsident Günter Verheugen; vgl. Heinrich Schneider: Religionsfreiheit, Kulturkampf, oder was sonst?, in: Friedrich Gleißner/Hanspeter Ruedl/ Heinrich Schneider/Ludwig Schwarz (Hrsg.): Religion im öffentlichen Raum, Wien/Köln/Weimar 2007, S. 11ff., hier S. 17-18.

4 „Im Namen der Allerheiligsten Dreifaltigkeit...“. Religionsangelegenheiten ansprechende Auszüge aus mitgliedstaatlichen Verfassungstexten finden sich im Anhang des Buches von Joseph H. H. Weiler: Ein christliches Europa - Erkundungsgänge. Mit einem Vorwort von Ernst-Wolfgang Böckenförde, Salzburg/München 2004, S. 154ff. Auch das, wenn man es so nennen kann, Grunddokument des „westfälischen Systems“, mit dem sowohl das Miteinander souveräner Staaten wie die Plurikonfessionalität Europas besiegelt wurde, der Friedensvertrag zu Münster vom 24. Oktober 1648, beginnt - wie viele Friedensverträge der europäischen Neuzeit - mit dem Satz: „Im Namen der allerheiligsten und unteilbaren Dreifaltigkeit, Amen.“ 
eines Teils des sich als Gemeinwesen konstituierenden Volkes; ein Beispiel hierfür ist die Präambel der polnischen Verfassung. Bekanntlich gab es Bestrebungen, einen ähnlichen Text in den Verfassungsvertrag hineinzureklamieren. ${ }^{5}$

Eine andere, auf das Wort ,Gott' verzichtende Option, für die sich auch Mitgliedstaaten einsetzten, wäre ein ausdrücklicher Hinweis auf christliche (oder auch auf jüdische und christliche) Komponenten des europäischen Erbes gewesen. Auch dabei kann man an mehrere Varianten denken; zum Beispiel an eine - dem Anschein nach - bloße historische Tatsachenfeststellung: dass das Christentum eine (oder in religiöser Perspektive sogar: die bestimmende) gestaltende Kraft Europas gewesen ist; womöglich auch unter Hinweis auf seine jüdischen Wurzeln. Engagierte Christen mochten sich sogar die Aussage gewünscht haben, dass dieses Erbe auch heute noch die Identität Europas mitprägt. Gewiss kann, wer dafür eintritt, die anderen, nichtreligiösen Komponenten des europäischen Geisteserbes nicht einfach leugnen, etwa die Vermächtnisse der griechischen und römischen Antike, des Humanismus oder der Aufklärung. Eine Variante wäre die ausdrückliche Nebeneinanderstellung solcher Kräfte und Vorgänge; eine schwächere war die pauschale Bezugnahme auf das religiöse, geistige (spirituelle) und sittliche Erbe der Völker Europas (wie etwa in der Präambel zur Grundrechtecharta), oder auf die kulturellen, religiösen und humanistischen Überlieferungen (vergleiche die Präambel zum Verfassungsvertrag). Dabei gab und gibt es heikle Empfindlichkeiten, manche haben sich sogar in sinnungetreuen Übersetzungen niedergeschlagen. ${ }^{6}$ Engagierte Christen bekundeten Unmut darüber, dass die ausdrückliche Erwähnung des ,christlichen Erbes' unterblieb - dieses sei doch „ein Faktum, das nur Ignoranten zu leugnen imstande sind“. ${ }^{7}$ Freilich geht es in der Textgattung ,Verfassungspräambeln' kaum um historische ,Erkenntnisse', vielmehr so gut wie stets um ,Bekenntnisse‘. Wird aus einem Universum (oder Pluriversum) historischer Fakten eine Auswahl getroffen, dann spiegelt sich in ihr das Leitbild des Gemeinwesens wider; das Ringen darum, was genannt oder verschwiegen wird, kann Kulturkampfcharakter haben. ${ }^{8}$

Ergänzend oder alternativ zur Umschreibung des in die ,religion civile" eingehenden historischen Erbes käme ein Bekenntnis zu identitätsstiftenden ,Werten ' infrage, ${ }^{9}$ weil dies den Verzicht auf ,Historisches' einschließt und daher dem Bekenntnis einen allgemeineren Charakter gibt. So könnten auch ,christliche Werte', oder, noch weiter gefasst, ,religiöse' genannt werden, sei es unter Verwendung entsprechender Adjektive, oder so, dass die religiöse (christliche) Herkunft der betreffenden Begriffe mehr oder weniger offenkundig ist. ${ }^{10}$

5 Vgl. die Wiedergabe bei Weiler: Ein christliches Europa, 2004, S. 156f.: „Wir, die Polnische Nation, alle Bürger der Republik, sowohl jene die an Gott als die Quelle der Wahrheit, der Gerechtigkeit, des Guten und Schönen glauben, als auch jene, die einen solchen Glauben nicht teilen, diese universalen Werte aber als aus andren Quellen entspringend achten, gleich in Rechten und Pflichten,..., errichten Polen...".

6 In der Präambel der Grundrechtecharta wurde die französische Formel ,patrimoine spirituel et morale“ (im Englischen „spiritual und moral heritage“) ins Deutsche in die Wendung vom „geistig-religiösen und sittlichen Erbe“ übersetzt; Frankreich war nicht bereit gewesen, den Ausdruck „,réligieuse“ in ,seinem“ Text zu dulden; siehe dazu Sven Hölscheidt/Eva Mund: Religionen und Kirchen im europäischen Verfassungsverbund, in: Europarecht 6/2003, S. 1083-1094, hier S. 1087f.

7 Michael H. Weninger: Europa ohne Gott? Die Europäische Union und der Dialog mit den Religionen, Kirchen und Weltanschauungsgemeinschaften, Baden-Baden 2007, S. 225, unter Hinweis auf kritische Äußerungen hoher vatikanischer Würdenträger.

8 Vgl. dazu Peter Häberle: Europäische Verfassungslehre, Baden-Baden 2001/2002, S. 273ff.; dort auch weitere Literaturhinweise zum Gegenstand.

9 Dass die Rede von „Werten“ und von der „Europäischen Wertegemeinschaft“ bei Weitem problematischer ist als ihre weite Verbreitung nahelegt, wird oft verdrängt; vgl. statt vieler: Heinrich Schneider: Die Europäische Union als Wertegemeinschaft auf der Suche nach sich selbst, in: Die Union - Vierteljahresschrift für Integrationsfragen (Wien) 1/2000, S. 11ff.; Robert Spaemann: Europa - Wertegemeinschaft oder Rechtsordnung, in: Transit - Europäische Revue, Nr. 21 (Sommer 2001), S. 172ff.

10 Vgl. den Verweis auf die Menschenwürde und auf die Zentralität der Person in der Präambel zur Grundrechtecharta. 
Worum ging es im Tauziehen um die Einbeziehung solcher Bekenntnisse in eine Verfassungs- oder Vertragspräambel? Aufs Erste scheint das klar: den jeweils Engagiertesten um die Durchsetzung des Anspruchs auf verbindliche Fixierung entweder der Christlichkeit oder der Säkularität des Gemeinwesens; sofern weniger weitgehende Positionen verfochten wurden, immerhin um die Legitimität öffentlicher Bekundung religiöser Überzeugungen und auf ihnen beruhender Ansprüche, auch mit rechtlichen Konsequenzen. ${ }^{11}$ Kirchen und Kirchengegner schienen einander gegenüberzustehen.

Aber man sollte es sich nicht zu einfach machen. Wer die Konstellation der Beweggründe rekonstruieren will, sollte sich zur Vermeidung von Kurzschlüssen und Engführungen wohl erst das breite Feld der Möglichkeiten vergegenwärtigen.

Ein Analytiker hat gemeint, die Angelegenheit habe ihren Hauptgrund im Bestreben der Mitgliedstaaten gehabt, ihre jeweiligen Politikvorstellungen auf die Ebene der Union „hochzuladen" ${ }^{12}$ um Dissonanzen zwischen nationalem und europäischem ordre public (und den entsprechenden Bestandteilen der politischen Kultur) möglichst gering zu halten. Die Vermutung hat einiges für sich, dass die europapolitischen Akteure - Regierungen und andere Konventsmitglieder in erster Linie - in ihrer Einstellung zu entsprechenden Forderungen davon bestimmt wurden, wie sie die richtige oder wünschenswerte Ordnung der Dinge zu sehen gewohnt sind, vor allem aufgrund der ihnen vom eigenen Land her vertrauten Gegebenheiten: Politiker aus Paris, der Hauptstadt jener Republik, die sich im ersten Verfassungsartikel von 1958 als ,laizistische“ deklariert hat, werden in die Diskussion andere Vorstellungen mitbringen, als solche aus La Valletta, deren Verfassung ,die Römische Katholische Apostolische Religion“ als ,die Religion Maltas“ bezeichnet. ${ }^{13}$ Nur Derartiges nachzuprüfen wäre jedoch zu einfach. Erstens könnte man fragen, ob und inwieweit dabei (auch) eine womöglich nur wenig reflektierte Tendenz der Konventualen im Umgang mit kognitiven und evaluativen Dissonanzen wirksam ist, zugunsten ihrer Verdrängung oder Überbrückung; das dürfte hier nicht besonders fruchtbar sein, weil die kirchenfreundlichen Positionen so markant von ihren Vertretern ins Spiel gebracht wurden, dass die Adressaten sich damit auseinandersetzen mussten.

Bedachtsam präsentierte Stellungnahmen - im Konvent wie in der Regierungskonferenz - konnten indessen auf sehr unterschiedlichen Beweggründen beruhen:

Dem Versuch, das heimische religionspolitische Regime auf die europäische Ebene zu übertragen, konnten grundlegende religionspolitische Überzeugungen zugrunde liegen. ${ }^{14}$

11 Die Europäische Menschenrechtskonvention ermächtigt, wie der Straßburger Gerichtshof festgestellt hat, die Staaten, für Mitglieder von Religionsgemeinschaften Ausnahmen von der Pflicht zur Befolgung der allgemeinen Gesetze vorzusehen, wenn eine religiös-pflichtgemäße Handlungsweise mit den entsprechenden gesetzlichen Bestimmungen in Widerspruch steht - nur dann allerdings, wenn die Ausnahmeregelung nicht die öffentliche Sicherheit und Ordnung, die Gesundheit, die Moral oder Rechte und Freiheiten anderer beeinträchtigt. Solche Ausnahmeregelungen gibt es in einer ganzen Reihe von Ländern - für Christen, für Juden, zuweilen auch für Muslime, und für andere. Dabei handelt es sich jedoch um Konsequenzen des Grundrechts auf Religionsfreiheit, dessen Verbindlichkeit von Bestimmungen wie den hier betrachteten nicht abhängt.

12 So Gregor Waschinski: Gott in die Verfassung? Religion und Kompatibilität in der Europäischen Union, Baden-Baden 2007, S. 57 und öfter.

13 Art. 2 Abs. 1 der Verfassung; die folgenden Absätze lauten: „Die Autoritäten der Römischen Katholischen Apostolischen Kirche haben das Recht und die Pflicht zu lehren, welche Prinzipien richtig und welche falsch sind.“ (Abs. 2); ,Religiöser Unterricht des Römischen Katholischen Apostolischen Glaubens soll in allen staatlichen Schulen Teil der verpflichtenden Erziehung sein.“(Abs. 3). Nach Weiler: Ein christliches Europa, 2004, S. 156.

14 Analogerweise etwa so, wie von deutscher Seite bei den Verhandlungen um die Römischen Verträge und ihrer Vorbereitung (im Spaak-Ausschuss) versucht wurde, die heimischen Vorstellungen von Sozialer Marktwirtschaft für die EWG so weit wie möglich verbindlich zu machen; darauf hat Hans von der Groeben als Beteiligter immer wieder hingewiesen. 
Solche Überzeugungen konnten aber auch dazu bewegen, für die europäische Ebene, aus welchen Gründen immer, ein markant anderes Regime als das im eigenen Land etablierte für gut zu halten. ${ }^{15}$ Von vornherein war auch vermutbar, dass parteipolitische Positionen die Einstellung der Akteure prägten, so dass zum Beispiel Christdemokraten den Wünschen der Kirchen freundlich gegenüberstanden, Liberale und kirchenkritisch eingestellte Sozialisten nicht.

An der Grenze zwischen im strikten Sinn religionspolitischen und fundamentalpolitischen (gewissermaßen die Sinnbestimmung der Politik betreffenden) Beweggründen könnte man die Überlegung verorten, dass eine - aus Gründen der Tradition naheliegenderweise in religiöser Sprache artikulierte - Transzendenz-Bezugnahme geeignet wäre, totalitären Bestrebungen wenigstens ideell einen Riegel vorzuschieben; etwa in der Linie des Arguments: die Würde des Menschen ist nur dann unantastbar, wenn es eine Begründung für sie gibt, die menschlicher Macht und Manipulation entzogen ist, sodass der Politik Schranken ihrer Verfügungsbefugnis gesetzt sind. ${ }^{16}$

Im Ringen um die Religionsreferenzen in der Verfassungspräambel mochten aber auch Überlegungen im Spiel gewesen sein, die weder einen direkt religions- noch einen fundamentalpolitischen Bezug hatten. Möglicherweise konnte es Befürwortern um eine Aufwertung ,ideeller', meta-ökonomischer Sinngehalte des ,Projekts Europa' zu tun sein, beziehungsweise - in gleicher Richtung, aber mit anderer Fokussierung - um die Relativierung des von manchen Kritikern der traditionellen Integrationspolitik - mit Recht oder Unrecht zugeschriebenen „Ökonomismus“. ${ }^{17}$

Nicht identisch damit, aber im Effekt wahlverwandt wäre die (mögliche) Absicht, jede inhaltliche Anreicherung der Vertrags- oder Verfassungspräambel, und damit auch eine Bezugnahme auf spirituelle oder religiöse Sinngehalte, als einen Beitrag zur prinzipiellen Aufwertung der Europäischen Union zu befürworten. Jeder Beitrag hierzu würde die Union deutlicher als ein Gebilde charakterisieren, das die Zweckverbandskonzeption der Frühzeit hinter sich lässt (wiewohl die Wirtschaftsintegration, primär durch die Fusion nationaler Märkte, ursprünglich der Kern des Vergemeinschaftsprojekts, ohnehin nicht mehr der einzige Zweck des Projekts ist). Der Charakter einer ,Polity', eines Gemeinwesens, das um der sinnvollen menschlichen Lebensführung willen eingerichtet ist, nicht nur zur Verbesserung von Bedingungen des Überlebens, würde betont. ${ }^{18}$ Zugleich würde damit auch der Verfassungscharakter der rechtlichen Grundordnung der Union bekräftigt. Zudem wäre ein Verweis auf religiöse Bezüge, etwa nach dem Vorbild der polnischen Präambel, ein Indiz für

15 Klarerweise wird man Derartiges etwa überzeugten Liberalen oder religiös Freisinnigen (Atheisten) aus Ländern mit einem starken kirchlichen Einfluss auf das öffentliche Leben unterstellen können.

16 Verfechter der Einfügung religiöser Wendungen in die Verfassungspräambel haben gelegentlich so argumentiert, um auch Andersdenkende für ihr Anliegen zu gewinnen. Dies schließt aber nicht aus, dass auch ein ,philosophischer Glaube“ (im Sinn z.B. von weiland Karl Jaspers) sich berufen fühlen mag, der Politik klar zu machen, dass ihre Sache allenfalls das Reich der ,vorletzten Dinge“ umfasst, weil nur auf Basis dieser Sicht dem Fanatismus und dem Totalitarismus die Legitimität mit gutem Grund verweigert werden kann. Die Logik des Arguments geht freilich verloren, wenn religiöse Fundamentalisten oder Integralisten ihrerseits meinen, Politik direkt auf die ,letzten Dinge“ hin ausrichten zu sollen.

17 Vor einem „Ökonomismus“, der Gemeinwohlerfordernisse zugunsten der Orientierung an Erfordernissen der Marktlogik vernachlässigt oder unternehmerischen Gewinnkalkulationen unterordnet, warnte z.B. Papst Johannes Paul II.; siehe Schneider: Die Europäische Einigung als Thema der Katholischen Kirche, 2003, S. 96f.; Häberle (Europäische Verfassungslehre, 2001/2002, S. 522) meint, dass die Einfügung von Ansätzen zu einem „europäischen Religionsverfassungsrecht“ in das Unionsrecht einen „glücklichen Gegenakzent zum bislang (über)betonten ,Europa der Wirtschaft" setzt".

18 Dies erinnert an den aristotelischen Satz, dass die pólis zur Sicherung des (Über-) Lebens (,zên ") entstand, aber um des guten, menschenwürdigen Lebens (,eu zên') willen besteht, oder an den neuzeitlichen, dass sich über dem Reich der Notwendigkeit das Reich der Freiheit konstituiert. 
eine verstärkte Eigenständigkeit der Union im Verhältnis zu den Mitgliedstaaten, von denen ja einige etwas Derartiges als mit ihrer eigenen Laizität unvereinbar betrachten; eine entsprechende Wendung würde den Charakter der verfassten Europäischen Union als einer den bloßen Staatenverbund transzendierenden Union (auch), von Bürgern' (nämlich von gläubigen und ungläubigen) akzentuieren.

Mit anderen Worten: Das Ringen um die Ausstattung der Präambel mit einem spirituellen oder religiösen Bezug, gar mit einem Hinweis auf Gott, war auch ein Element des Ringens um die Annäherung der Union an das, was man früher (bis in die Ära Delors) eine Föderation und später noch eine Politische Union genannt hat, und ein Moment der relativen Stärkung der „sozialintegrativen“ Dimension des Staaten- und Bürgerverbundes im Verhältnis zur - im Sinne von Habermas - ,systemintegrativen“ Zusammenführung von Märkten und politisch-administrativen Strukturen und Mechanismen. Das heißt umgekehrt: Von Gegnern der Aufwertung der Union zu einer ,Polity', vor allem von jenen, die in der Integrationsvertiefung und in der Stärkung der normativen Grundordnung der Europäischen Union (also etwa in der emphatischen Aufwertung der Verträge zur, Verfassung ') eine Tendenz hin zu einem ,Superstaat' sehen, konnte man schon deshalb erwarten, dass sie sich der Anreicherung der Präambel widersetzen würden.

Auch in einer weiteren Dimension konnte der Gedanke an die Finalität der Union eine Rolle spielen: was die Grenzen ihrer Ausdehnung betrifft. Ein ausdrücklicher Hinweis auf die christliche Basis der europäischen Identität hätte eine Distanzierung nichtchristlicher Beitrittskandidaten wie der Türkei bedeutet; andererseits würde ein nicht ausdrücklich als christlich oder jüdisch-christlich akzentuierter, Gottesbezug ' Muslime einschließen.

Andere taktische Motive mochten zusätzlich im Spiel sein. In Polen beispielsweise hatten sich katholisch-konservative Kräfte (wie Pater Tadeusz Rydzyk und sein Sender ,Radio Maryja') konsequent gegen die ungebremste Integration des Landes in das westlich-liberale, in ihrer Sicht die Tradition und die Moral zersetzende Europa von Brüssel gewandt und mit ihrer Darstellung der Dinge die Frustration entsprechend gesinnter Teile der Bevölkerung vorangetrieben. So mussten politische Akteure, denen an der Schwächung antieuropäischer Tendenzen in der Gesellschaft gelegen war (um der Erhaltung der inneren Einigkeit des Volkes willen, aber auch um einer Isolierung Polens in der Union entgegenzuwirken) an einer Selbstpositionierung der Europäischen Union interessiert sein, die dem liberalen und fast , antichristlichen‘ Image Brüssels entgegengestellt werden konnte. Das Engagement polnischer Politiker für den Gottesbezug und für die Namhaftmachung christlicher Traditionen und Werte in der Verfassungspräambel hatte daher vermutlich auch solche Beweggründe.

Schließlich muss man auch noch mit mannigfachen anderen taktischen Beweggründen des Eintretens für oder gegen die Präambelhinweise rechnen (und auch für oder gegen die Kirchen und Religionsgemeinschaften aufwertenden Vertragsbestimmungen). Rücksichtnahmen auf innenpolitische Konstellationen können dabei eine Rolle spielen (nicht nur parteipolitische, sondern auch solche, die sich auf die religiöse und weltanschauliche Konstellation in der Gesellschaft beziehen), aber auch Verhandlungskonstellationen in der Regierungskonferenz selbst (gemäß der Regel, do ut des' wird durch die Übernahme oder die Abwertung einer Detailposition ein Entgegenkommen anderer Partner in einer anderen Detailfrage eingeworben).

Das alles sind Hypothesen; man sollte sie sich aber möglichst ohne Vereinfachungen vor Augen führen, bevor man zu ermitteln sucht, welche Motiv- und Kräftekonstellationen mit welcher Einflussstärke tatsächlich wirksam waren. 


\section{Das andere Anliegen: der ,Kirchenartikel}

Die Sachwalter kirchlicher Anliegen merkten im Gang der Auseinandersetzung, dass in Bezug auf die Präambel ihre Hoffnungen enttäuscht werden würden; schon deshalb, weil das klare französische Nein zu Formeln, die mit dem Prinzip der Laizität in Spannung standen, außer Zweifel stand. So konzentrierten sie sich auf das zweite religionspolitisch bedeutsame Projekt.

Das war richtig; vermutlich war das Gewicht der religionsbezogenen Präambelwendungen ohnehin überschätzt worden: Welche handfeste Bedeutung eine Verfassungspräambel hat, hängt dort, wo es Verfassungsgerichte gibt, von deren Judikatur ab. Ob der Europäische Gerichtshof aus den religionsbezogenen Formulierungen viel herausgeholt (oder in sie hineingelegt) hätte, ist höchst zweifelhaft, und ebenso auch die andere Frage, ob die betreffenden Wendungen den Gerichtshof bei der Rechtsprechung zu verbindlichen Vertrags- oder Verfassungsartikeln substanziell beeinflusst hätten. Von solchen Folgen abgesehen wird jemand, der sein Handeln an christlichen oder anderen Glaubenstraditionen und -überzeugungen ausrichtet, dies wohl auch dann tun, wenn sich die EU-Vertragspräambel von Lissabon dazu ausschweigt; wer dazu eine ganz andere Einstellung hat, wird diese kaum dadurch ändern, weil eine europäische Verfassungspräambel ihm das nahelegt.

Die schließlich im Vertrag von Lissabon als Artikel 17 in den, Vertrag über die Arbeitsweise der Europäischen Union“ (AEUV) aufgenommene Bestimmung ist in der Tat für die Kirchen wichtiger als die Realisierung ihrer Präambel-Wünsche.

,Für die Kirchen " - um ihre Wünsche ging es übrigens in den entsprechenden Auseinandersetzungen primär und hauptsächlich; andere religiöse Vereinigungen und insbesondere die (nichtreligiösen) Weltanschauungsgemeinschaften erhielten die gleiche Zusicherung, weil sonst die Privilegierung einer Religion (oder einiger von vielen Religionen) vorgenommen worden wäre, und dies hätte die Chancen für die Annahme des Artikels minimiert. ${ }^{19}$

Schon in der Schlussakte zum Vertrag von Amsterdam findet sich die Erklärung Nr. 11, derzufolge die Union den Status von Kirchen, religiösen Vereinigungen und Gemeinschaften und in gleicher Weise den von weltanschaulichen Gemeinschaften achtet, wie ihn die Rechtsvorschriften der Mitgliedstaaten festlegen. Dass nicht nur dies in den Konventsentwurf des Verfassungsvertrages übernommen, sondern auch noch durch die eingangs erwähnte Klausel über den mit Kirchen, religiösen und weltanschaulichen Gemeinschaften ,,in Anerkennung ihrer Identität und ihres besonderen Beitrags“ zu führenden „offenen, transparenten und regelmäßigen Dialog“ ergänzt wurde, nimmt dem Vertragsartikel seine Selbstverständlichkeit.

Die Verpflichtung der Union, den mitgliedstaatlich fixierten Status von Kirchen und gleichgestellten Vereinigungen zu achten, ist der Sache nach eine Spezialklausel im Rahmen der Verpflichtung der Union zur Achtung der nationalen Identität der Mitgliedstaaten, wie sie schon als Artikel 6 Absatz 3 in den EU-Vertrag von Amsterdam eingefügt worden war, weil es Besorgnisse gab, die Integration könnte die nationale Identität der Mitgliedstaaten infrage stellen.

19 Moderne Grundrechtekataloge sprechen von „Gedanken-, Gewissens- und Religionsfreiheit“ (siehe die Europäische Grundrechtecharta, aber auch schon den Internationalen Pakt über bürgerliche und politische Rechte von 1966); Religionen und Weltanschauungen sind hierbei gleichgestellt, und neben der „positiven“ gibt es auch die ,,negative Religionsfreiheit“, also das Recht, religiöse Überzeugungen und Praktiken abzulehnen und für diese Ablehnung öffentlich einzutreten. Als Kommissionspräsident Barroso, sozusagen im Vorgriff auf die Bestimmung über den Dialog, Vertreter christlicher Kirchen zu einem Meinungsaustausch einlud, aber entsprechende Zusammenkünfte mit religionskritischen Verbandsvertretern erst danach anberaumen wollte, musste er sich harsche Kritik im Europäischen Parlament anhören, vgl. Schneider: Religionsfreiheit, Kulturkampf, oder was sonst?, 2007, S. 14f. 
Gute Gründe sprechen dafür, nationale Religionsverfassungsregimes als Elemente der nationalen Identität zu betrachten, im Hinblick auf die Eigenart sowohl der Rechtsordnung wie des Selbstverständnisses und der politischen Kultur. ${ }^{20}$ Zum Selbstverständnis der Französischen Republik gehört zweifellos die ,Laïcité‘. Aber kann man andererseits sagen, dass der öffentlich-rechtliche Status der großen Religionsgemeinschaften zur Identität der Bundesrepublik oder der Republik Österreich gehört? Der ,Kirchenartikel' des Vertrags von Lissabon (Artikel 17 AEUV) kann entsprechende Zweifel beheben. Er spricht dem Religionsverfassungsregime eine besondere Respektabilität zu. ${ }^{21}$

Dies war im Kontext der Unionsrechtsordnung nicht selbstverständlich; die Autoren der Formulierung hätten sonst kaum Anlass gehabt, auf die „Anerkennung“ der „Identität“ und des „,besonderen Beitrags“ dieser Dialogpartner (gemeint ist wohl der Beitrag zur Förderung der Ziele der Europäischen Union und zu ihrer Verwirklichung) hinzuweisen. Die NichtSelbstverständlichkeit ergibt sich daraus, dass es keine genuine Religions- und Kirchenpolitik der Europäischen Union gibt; die entsprechende Politik bleibt den Mitgliedstaaten vorbehalten. Wenn es um Rechtsvorschriften geht, die besondere Interessen und Anliegen der Kirchen berühren (wie etwa das Verbot der Diskriminierung aus religiösen Gründen), wäre es ja auch möglich gewesen, von der Bestimmung über die Anhörung der Betroffenen (Artikel I-47 Absatz 3 des Konventsentwurfs für den VVE, im Vertrag von Lissabon Artikel 11 Absatz 3 EUV) extensiv Gebrauch zu machen. Die Kirchen als mit den Bestimmungen zur „partizipativen Demokratie“ (Artikel I-47 VVE) beziehungsweise über die ,demokratischen Grundsätze“ (im Vertrag von Lissabon Artikel 9-12 EUV) mitgemeint zu betrachten, das heißt als Partner des Dialogs der Unionsorgane ,mit den repräsentativen Verbänden und der Zivilgesellschaft“, war problematisch; die Kirchen selbst wollten nicht einfach der „Zivilgesellschaft" zugerechnet werden (unter Berufung auf ihren Charakter als Einrichtungen nicht nur „von dieser Welt“). ${ }^{22}$ Kirchengegner bestreiten nicht selten die Repräsentativität der Religionsgemeinschaften in demokratiepolitischer Perspektive; insbesondere den zuweilen erhobenen Anspruch, alle Mitglieder gegenüber den Regierenden zu vertreten - wer Religion als Privatsache betrachtet, wird Religionsgemeinschaften kaum ein politisches Mandat zuerkennen.

Vor allem die großen Kirchen nehmen aufgrund ihres Selbstverständnisses jedenfalls ein Mitspracherecht in öffentlichen Angelegenheiten in Anspruch. Auf katholischer Seite hat

20 Vgl. die Kommentierung der Bestimmungen des geltenden Vertrags (Stand: Nizza) von Meinhard Hilf und Frank Schorkopf, in: Eberhard Grabitz/Meinhard Hilf: Das Recht der Europäischen Union, München 2004, hier zu Art. 6 Abs. 3 EUV, Rdn. $72 \mathrm{ff}$.

21 Das kann man sich klar machen, wenn man überlegt, ob auch eine vergleichbare Fixierung der Pflicht der Union, den öffentlich-rechtlichen Status der ,Kammern` zur Gewährleistung der nationalen Identität (nämlich der legitimitätssichernden Eigenart des mitgliedstaatlichen ordre public) zu respektieren, vorstellbar wäre. Im Vorfeld des Beitritts des - wie man damals gern sagte - , Kammerstaates' Österreich war die Frage nach der Sicherung der Grundlagen der österreichischen Sozialpartnerschaft ein ernsthaft erörtertes Thema der Innen- und der Europapolitik.

22 Vor allem von katholischer Seite wurde die Einordnung der Kirche selbst in die Zivilgesellschaft abgelehnt; wiewohl man für kirchliche und kirchennahe Vereinigungen die Verortung in ihr bejaht; im evangelischen Bereich ist die Ablehnung heute weniger ausgeprägt. Indessen ist - nicht überall in Europa - zuweilen die Andersheit der Kirchen im Vergleich zu zivilgesellschaftlichen Verbänden auch von, weltlicher ' Seite unmissverständlich anerkannt worden; siehe z.B. die Aussage von Bundeskanzler Willy Brandt (SPD) in seiner Regierungserklärung am 18. Januar 1973 über die Kirchen: „Wir betrachten sie nicht als eine Gruppe unter den vielen der pluralistischen Gesellschaft und wollen ihre[n] Repräsentanten darum auch nicht als Vertreter[n] bloßer Gruppeninteressen begegnen. Wir meinen im Gegenteil, daß die Kirchen in ihrer notwendigen geistigen Wirkung um so stärker sind, je unabhängiger sie sich von überkommenen sozialen oder parteilichen Bindungen machen. Im Zeichen deutlicher Freiheit wünschen wir die Partnerschaft" (zit. nach: Bulletin des Presse- und Informationsamtes der Bundesregierung vom 19.01.1973, Nr. 6, S. 56). 
man die Aufgabe der Kirche als „Aktivierung des öffentlichen Gewissens im Dienste des Gemeinwohls“ zur „Weckung der grundlegenden Wahrheits- und Wertüberzeugungen“ bezeichnet, wobei besonders die Laien zum Engagement berufen seien. ${ }^{23} \mathrm{Im}$ evangelischen Sprachduktus nimmt die Kirche einen „Öffentlichkeitsauftrag“ wahr; in der Ausübung ihres „Wächteramtes“ will sie vor allem auf die Grenzen menschlicher Machtausübung und auf Missbräuche der Macht und auf Defizite bei der Verwirklichung von Freiheit und Gerechtigkeit aufmerksam machen. Die Religionsfreiheit schließe auch das Menschenrecht ein, den herrschenden Mächten im Einklang mit der Glaubensüberzeugung ins Gewissen zu reden. ${ }^{24}$

Befürworter der besonderen Würdigung der Kirchen argumentieren: die Kirchen ständen als „Institutionen geistlicher Daseinsvorsorge“ im Dienste öffentlicher Bedürfnisse, sodass ein öffentlich-rechtlicher Status um ihrer Beiträge zum Gemeinwohl willen gerechtfertigt sei. ${ }^{25}$ So weit geht der ,Kirchenartikel“ des Vertrags von Lissabon nicht, aber hinter der „Anerkennung des besonderen Beitrags“" stehen gewiss auch Erwägungen, die in die Richtung der eben referierten gehen - nicht nur der Gedanke an die Honorierung positiver Impulse der Kirchen zur europäischen Einigung.

Klarerweise werden Vorstellungen, die vom Selbstverständnis der Kirchen abgeleitet sind, oder auch solche, die von einer anthropologisch gegebenen Religiosität des Menschen ausgehen (oder zumindest von einer Anlage dazu, oder gar von einem ,religiösen Existential"), von Andersdenkenden abgelehnt; dass diese ,kirchliche Privilegien" heftig kritisiert und auch gegen den ,Kirchenartikel' interveniert haben, war nicht verwunderlich. Schließlich kam es der Ausgewogenheit zuliebe zur Zuerkennung der zunächst von den Kirchen für sich reklamierten Qualifikationen und Zusagen auch an die ,weltanschaulichen Gemeinschaften" (nicht nur an andere Religionsgemeinschaften).

23 So, die Positionen des II. Vatikanischen Konzils vorwegnehmend und ihnen den Weg weisend, etwa Franz Kardinal König: Kirche, Staat, Gesellschaft - Die Funktionen der Kirche für das Gemeinwesen von heute, in: Wort und Wahrheit - Monatsschrift für Religion und Kultur 16. Jg. 2/1961, S. $91 \mathrm{ff}$. Das Konzil hat dann - unterschieden von autoritativen Aussagen und Aktionen der leitenden Amtsträger (des Papstes, der Bischöfe) noch eine weitere Unterscheidung betont, nämlich die zwischen solchen Unternehmungen, die Christen als einzelne oder im Verbund im eigenen Namen als Staatsbürger in die Hand nehmen, und jenen, die sie ,im Namen der Kirche zusammen mit ihren Hirten“ vollziehen (Pastoralkonstitution „Gaudium et Spes“, 1965, Abschnitt 76). Die eigene Sendung der Kirche sei religiös, nicht politisch, aber aus dieser Sendung erfließe der Auftrag und die Kraft, der menschlichen Gemeinschaft behilflich zu sein (ebenda Abschnitt 42); und für die weltlichen Dinge (die Politik eingeschlossen) seien eigentlich, wenn auch nicht ausschließlich, die Laien zuständig; treten dabei legitime Meinungsverschiedenheiten über die richtige Politik auf, solle niemand die Autorität der Kirche ausschließlich für sich und die eigene Meinung in Anspruch nehmen (ebenda Abschnitt 43). Angesichts dessen ist es auffällig, dass in europapolitischen Fragen katholische Standpunkte und Wünsche in erster Linie von geistlichen Amtsträgern (namentlich von Päpsten und von der ,Kommission der Bischofskonferenzen der Europäischen Gemeinschaft' geltend gemacht wurden, wobei diese in der Auseinandersetzung um den Verfassungsvertrag auf die ökumenische Abstimmung mit der ,Konferenz Europäischer Kirchen “ Bedacht nahm).

24 Vgl. Dietrich Pirson: Öffentlichkeitsanspruch der Kirche, in: Roman Herzog/Hermann Kunst/Klaus Schlaich/ Wilhelm Schneemelcher (Hrsg.): Evangelisches Staatslexikon, 3. Aufl., Stuttgart 1987, Band 2, Sp. 2278ff.; Rüdiger Schloz: Religionsfreiheit, in: Herzog/Kunst/Schlaich/Schneemelcher: Evangelisches Staatslexikon, 1987, Bd. 2, Sp. $2966 \mathrm{ff}$.

25 So z.B. Paul Mikat: Zur rechtlichen Bedeutung religiöser Interessen, in: Material zum Problem Kirche und Politik, Heft 33 der Materialien der Politischen Akademie Eichholz, Bonn 1975, S. 9ff., hier S. 15. Man kann dabei auch an die Frage denken, ,ob nicht auch der säkularisierte Staat letztlich aus jenen inneren Antrieben und Bindungskräften leben muß, die der religiöse Glaube seiner Bürger vermittelt" - weil sich die Freiheit im freiheitlichen Staat aus der „,moralischen Substanz des einzelnen“ reguliert (Ernst Wolfgang Böckenförde: Die Entstehung des Staates als Vorgang der Säkularisation, 1967; hier nach: Böckenförde: Staat - Gesellschaft Freiheit, Frankfurt am Main 1976, S. 42ff., hier S. 61, S. 60). Damit sind nicht gemeinnützige Leistungen der Kirchen im Bildungs- und Gesundheitswesen, in der Kulturpflege und dergleichen gemeint; vgl. Hans Maier: Dienste der Kirche am Staat - Entwurf einer Typologie, in: Die Verantwortung der Kirche für den Staat (Essener Gespräche, Band 25), Münster 1991, S. 5ff. 
Dies hat dem Vertragsartikel ein etwas eigenartiges Gepräge gegeben, nicht nur durch die Art der ,Dazwischenschiebung ' des zweiten Absatzes nach dem ersten und vor dem dritten, sondern auch weil die Gründe für die Abhebung der Kirchen und Religionsgemeinschaften von anderen Verbänden und zivilgesellschaftlichen Gruppierungen nicht auch für die irreligiösen unterstellt werden müssen. Gerechtfertigt ist der Einschub von Absatz 2 inhaltlich ohnehin. ${ }^{26}$

\section{Das Resultat}

Die Formulierung der Präambel und des sogenannten ,Kirchenartikels‘ (diese Bezeichnung ist tatsächlich unangemessen!) sind das Resultat eines Ringens, das sich vordergründig um mehrere für die Rechtsordnung der Union nicht unwichtige, aber scheinbar nur für bestimmte Akteure und ihre Anliegen wichtige Fragen drehte:

- Die Sicherung der religionsverfassungsrechtlichen Regelungen der Mitgliedstaaten, als ein Element der nationalen Identitätswahrung;

- die ausdrückliche Affirmation religiöser, insbesondere christlicher Komponenten des geistigen Erbes Europas und damit um deren Anerkennung als heute noch bedeutsame Elemente der europäischen Identität; sowie

- die Zuerkennung eines besonderen Status an Kirchen, religiöse Vereinigungen und Körperschaften sowie an Weltanschauungsgemeinschaften, deren Beiträge eine Einbeziehung in den Prozess der Selbstverständigung Europas über seine geistige und politische Positionierung rechtfertigen. ${ }^{27}$

Nicht alles, was die christlichen Kirchen - als die eigentlichen Initiatoren - sich wünschten, haben sie erreicht, aber was schließlich in den Vertrag von Lissabon Eingang fand, war ihnen willkommen.

Die Beschränkung des Blicks auf das, was man die im Kontext der Europäischen Union ,Kirchen-, Religions- und Weltanschauungspolitik' nennen könnte, würde der Auseinandersetzung und ihren Implikationen jedoch nicht voll gerecht. Es ging dabei, wie schon angedeutet, auch um den Charakter der Union, um ihre weitere Ablösung von der bloßen Zweckverbandsnatur, um die Kräftigung der Qualität eines politischen Gemeinwesens. Es sieht

26 Das säkulare Gemeinwesen ist gegenüber religiösen und anderen Weltanschauungen neutral - dadurch unterscheidet es sich von einem ,säkularistischen“ ebenso wie von einem z.B. christlich oder muslimisch geprägten. Eine weitere Überlegung könnte davon ausgehen, dass Glaubensgemeinschaften einen besonderen Respekt verdienen, weil sie für die Gewissensfreiheit einstehen, der für das freiheitliche Gemeinwesen eine Schlüsselrolle zukommt; das Bundesverfassungsgericht hat z.B. erklärt, dass religiös motiviertes Wollen und Handeln besonderen Schutz verdient, weil es für die personale Identität konstitutiv ist; daher dürften Handlungen, die aus einer bestimmten Glaubenshaltung fließen, nicht ohne weiteres den Sanktionen unterworfen werden, die für sie vorgesehen sind, wenn sie nicht glaubensmäßig motiviert sind (BVerfGE 32, S. 98ff., hier S. 108); vgl. Stefan Huster: Religionsausübung und allgemeines Gesetz, in: Merkur 55. Jg. 625/2001, S. 424-429. Das hat das Gericht 2006 bekräftigt, wobei freilich unter Umständen auch eine Abwägung mit anderen Rechtsgütern nötig sei (siehe Süddeutsche Zeitung vom 21.06.2006, S. 6: Schulpflicht hat Vorrang). Sollte die besondere Würdigung von Gewissensüberzeugungen, die im Rahmen von Religionsgemeinschaften geweckt und gestärkt werden, allerdings bedeuten, dass die Gewissensfreiheit z.B. von Menschen, die sich einem ,philosophischen Glauben ' (etwa im Sinn von Karl Jaspers) verschrieben haben, weniger respektabel sei, dann wäre dies mit der Säkularität des modernen Gemeinwesens kaum zu vereinbaren; es liegt dann nahe, eine Diskriminierung von ,Weltanschauungsgemeinschaften', die sich um die Gewissensbildung ihrer Mitglieder bemühen, gegenüber religiösen Vereinigungen ebenfalls als unkorrekt anzusehen. (Ein „säkularistischer“ Staat könnte versucht sein, den gewissensbildenden Einflüssen der Kirchen Misstrauen entgegenzubringen und zum Beispiel den Atheismus und seine Propagierung gegenüber der religiösen Bekenntnisfreiheit privilegieren).

27 Die hier gewählte Formulierung ist etwas waghalsig. Im Vertragstext von Lissabon selbst bleibt unausgesprochen, welche Beiträge gemeint sind und man ist versucht zu meinen: solche zur europäischen Bewusstseinsbildung und damit zur ideellen Konsolidierung der Union. 
nicht so aus, als ob alle an der Erarbeitung, Durchsetzung oder Bekämpfung entsprechender Textpassagen Beteiligte dies stets vor Augen hatten; jedenfalls haben viele von ihnen das in den Diskussionen selbst kaum je zu erkennen gegeben. Im Zug der Konventsarbeit war dies nicht verwunderlich; waren doch die dort herrschende Stimmung und der Gesamtduktus der erstrebten Konstitutionalisierung dazu angetan, diese Qualitätsvertiefung als selbstverständlich erscheinen zu lassen. Als dann das Verfassungsprojekt als solches scheiterte, hatte die Substanz des nachmaligen Artikels 17 des ,Vertrags über die Arbeitsweise der Europäischen Union' bereits so klar Akzeptanz gefunden, dass ihr Erhalt nicht mehr infrage gestellt wurde. Das ist für die Verfechter der Sache erfreulich. Mehr wird für ihr Anliegen auf absehbare Zeit nicht zu erreichen sein. Ein neuer Konstitutionalisierungsschub steht nicht in Aussicht, und ohne ihn würde eine ,religionspolitische Stärkung ' der Union die mit dem erhofften Inkrafttreten des Vertrags von Lissabon zu etablierende Balance von System- und Sozialintegration infrage stellen. ${ }^{28}$

,Causa finita', möchte man sagen, wenigstens für die primärrechtliche Normierung und für die absehbare Zukunft. Wie sich die Umsetzung des Artikels 17 des, Vertrags über die Arbeitsweise der Europäischen Union“ und insbesondere seines dritten Absatzes darstellen wird, und ob sich daraus abermals Auseinandersetzungen ergeben, darauf werden zumindest einige der engagierten Akteure und Beobachter gespannt sein.

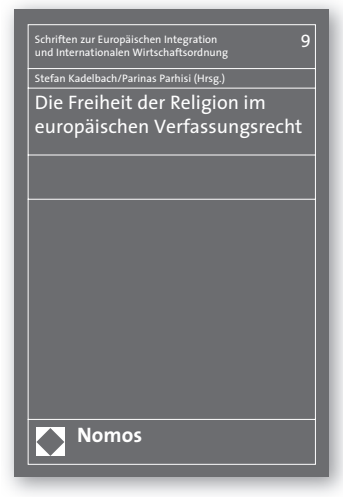

\section{Die Freiheit der Religion im europäischen Verfassungsrecht}

Herausgegeben von Stefan Kadelbach und Parinas Parhisi

2007, 194 S., brosch., 39,-€, ISBN 978-3-8329-2947-3 (Schriften zur Europäischen Integration und Internationalen Wirtschaftsordnung, Bd. 9)

Das vorliegende Buch enthält Beiträge zur Freiheit der Religion im europäischen Verfassungsleben, die das Thema aus verschiedenen wissenschaftlichen und praktischen Perspektiven untersuchen.

Bitte bestellen Sie bei Ihrer Buchhandlung oder bei Nomos | Telefon 07221/2104-37 | Fax -43 www.nomos.de | sabine.horn@nomos.de

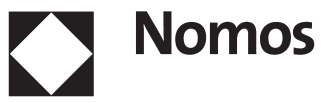

$28 \mathrm{Zu}$ den soeben verwendeten Begriffen vgl. Habermas: Theorie des kommunikativen Handelns, Band 1 und 2, 1981. Dass es Befürworter einer weiteren ,religionspolitischen Stärkung“ der Union gibt, wird im Blick auf Weninger (Europa ohne Gott?, 2007) deutlich. Zur Unwahrscheinlichkeit eines neuen Konstitutionalisierungsschubs vgl. z.B. Anne Faber: Die Weiterentwicklung der Europäischen Union: Vertiefung versus Erweiterung?, in: integration 2/2007, S. 103-116, vor allem S. 112-113; Klaus Hänsch: Ende gut - alles gut? Anmerkungen zum Reformvertrag, in: integration 4/2007, S. 499-502, vor allem S. 500. 